FELICJA KSIĘŻYK

\title{
Die Routiniertheit des Sprachgebrauchs am Beispiel kollokativer Verbindungen
}

Komunikacja językowa polega w większej mierze, niż dotąd zakładano, na zrutynizowanym użyciu języka. Jako typowy przykład wciąż powtarzanych sformułowań posłużyć mogą kolokacje. $\mathrm{Z}$ jednej strony współwystępowanie pewnych kombinacji wyrazowych uwarunkowane jest rzeczywistością pozajęzykową, z drugiej jednak kolokacje stanowią typowe dla danego języka, skonwencjonalizowane zjawiska $\mathrm{z}$ zakresu normy językowej. Artykuł przedstawia najistotniejsze cechy kolokacji w odniesieniu do języka polskiego i niemieckiego.

Die sprachliche Kommunikation beruht stärker als bisher angenommen auf einem routinemäßigen Sprachgebrauch. Als typisches Beispiel für wiederkehrende Formulierungen lassen sich Kollokationen anführen. Einerseits handelt es sich hierbei um Wortkombinationen, die durch die außersprachliche Realität bedingt sind, die andererseits aber auch einzelsprachspezifisch, konventionell sein können und Erscheinungen der Sprachnorm darstellen. Im vorliegenden Beitrag werden die wesentlichen Merkmale von Kollokationen diskutiert, und zwar in Bezug auf das Sprachenpaar Deutsch-Polnisch.

Language communication is more based on routine language use than it has been assumed so far. Collocations can serve as examples of constantly repeated phrases. On the one hand the co-occurrence of some word combinations is determined by extralingual reality but on the other hand collocations are typical and conventional for a given language phenomena belonging to the language norm. The current paper discusses the most essential criteria of collocations in reference to the German and Polish languages. 
Felicja Księżyk

\section{Kollokationen als Erscheinungen der Sprachnorm}

Bekanntlich wird nach Coseriu auf dem Gebiet der Sprache zwischen Rede (Parole) als der konkreten Realisierung der Sprachtechnik und den „drei sukzessiven Formalisierungsebenen dieser Technik: Norm, System und Typ der Sprache“ unterschieden (COSERIU 1970:38). Diese Unterschiede verdeutlicht Coseriu in Analogie zur Kunst:

Im Werk eines Künstlers kann eine konkrete Technik festgestellt werden, die in einem Einzelwerk sichtbar wird (entspricht der Ebene der Parole in der Sprache). Es kann festgestellt werden, was bei einem bestimmten Künstler in verschiedenen Werken immer wieder als Bevorzugung wiederkehrt, also bestimmte Motive oder bestimmte Lösungen technischer Probleme (entsprechen der Ebene der Norm in der Sprache). Weiter kann die spezielle Art der Technik des Künstlers betrachtet werden, aufgrund deren Bilder ihm zugeschrieben werden können, deren Maler nicht bekannt ist, und die es erlaubt, ihn nachzuahmen (was dem Sprachsystem entspricht). Schließlich können auch analoge Techniken für andere Kunstzweige wie Plastik, Architektur, Dichtung und Musik festgestellt werden. Alle diese in verschiedenen Kunstzweigen analogen Techniken können im Begriff ,Typus‘ zusammengefaßt werden. (COSERIU 1973:41f.)

Während das System „die Gesamtheit der möglichen Realisierungen [...] [umfasst, und zwar] auch das, was noch nicht realisiert worden ist, aber virtuell existiert, was ,möglich' ist, d.h. was nach den geltenden Regeln der Sprache gebildet werden kann“, bildet die Norm, ,all das, was ,existiert', was in der Sprachgemeinschaft realisiert ist“" (COSERIU 1970:40):

Die Norm enthält die üblichen, normalen, traditionellen, nicht unbedingt funktionellen Unterschiede der Technik der Rede. Das System dagegen enthält alles, was objektiv funktionell (distinktiv) ist. Das System ist also die Summe der Oppositionsstrukturen einer Sprache, während die Norm die Sprache als ,soziale Institution“ kennzeichnet. In der Norm findet sich das, was auf Grund des Systems bereits realisiert wurde, sie ist die formalisierte Gesamtheit der traditionellen Realisierungen. Das System dagegen enthält darüber hinaus auch die noch nicht verwirklichten Möglichkeiten einer Sprache, es ist die Gesamtheit der möglichen Realisierungen, die sich aufgrund der Regeln des Sprachsystems ergeben, auch wenn sie noch nicht verwirklicht sind. (COSERIU 1973:44)

Coseriu zufolge lassen sich Sprachen in solche einteilen, die eine Dominanz des Systems aufweisen, etwa Türkisch oder Ungarisch, und solche mit einer Dominanz der Norm, wozu er die indogermanischen, darunter die romanischen Sprachen und insbesondere das Französische zählt (vgl. COSERIU 1970:41). Die Auswirkung der Norm drücke sich darin aus, dass die vom System her möglichen Bedeutungen präzisiert und fixiert werden (vgl. COSERIU 1973:45). 
Zur Ebene der Norm gehören auch Kollokationen als ,durch keine Notwendigkeit rechtzufertigende lexikalische Syntagmen“ (COSERIU 1970:42). Es gibt nämlich keine systematischen Gründe dafür, weshalb einige Varianten des Systems nicht realisiert werden, vielmehr ist es durch die Sprachnorm bedingt, dass andere Varianten bevorzugt werden. Anhand folgender Belege wird verdeutlicht, dass für die Wahl bestimmter Varianten keine funktionelle Notwendigkeit vorliegt, dass allerdings die Norm nur bestimmte Kombinationen toleriert (vgl. COSERIU 1973:46). Derartige Präferenzen werden noch deutlicher im Sprachvergleich, wo es zum Teil Übereinstimmungen gibt:

\begin{tabular}{|l|l|l|l|}
\hline DEUTSCH & POLNISCH \\
\hline $\begin{array}{l}\text { normbedingte } \\
\text { Kombination / }\end{array}$ & $\begin{array}{l}\text { systembedingte / } \\
\text { alternative Aus- } \\
\text { drucksmöglichkeiten }\end{array}$ & $\begin{array}{l}\text { normbedingte } \\
\text { Kombination } \\
\text { / Kollokation }\end{array}$ & $\begin{array}{l}\text { systembedingte / } \\
\text { alternative Aus- } \\
\text { drucksmöglichkeiten }\end{array}$ \\
\hline $\begin{array}{l}\text { reines Gewis- } \\
\text { sen }\end{array}$ & $\begin{array}{l}\text { sauberes Gewissen / } \\
\text { reinliches Gewissen / } \\
\text { klares Gewissen / } \\
\text { unbeflecktes Gewissen }\end{array}$ & $\begin{array}{l}\text { czyste sumie- } \\
\text { nie }\end{array}$ & $\begin{array}{l}\text { porzadne sumienie / } \\
\text { sumienie bez zabru- } \\
\text { dzeń / } \\
\text { niezabrudzone sumie- } \\
\text { nie / } \\
\text { klarowne sumienie }\end{array}$ \\
\hline $\begin{array}{l}\text { gemischte } \\
\text { Gefühle }\end{array}$ & $\begin{array}{l}\text { zusammengesetzte } \\
\text { Gefühle / } \\
\text { gemixte Gefühle / } \\
\text { verschiedene Gefühle }\end{array}$ & $\begin{array}{l}\text { mieszane } \\
\text { uczucia }\end{array}$ & $\begin{array}{l}\text { niejednorodite uczucia / } \\
\text { cia / } \\
\text { ztożone uczucia }\end{array}$ \\
\hline $\begin{array}{l}\text { vollendete } \\
\text { Tatsachen }\end{array}$ & $\begin{array}{l}\text { abgeschlossene Tatsa- } \\
\text { chen / } \\
\text { beendete Tatsachen / } \\
\text { erledigte Tatsachen }\end{array}$ & $\begin{array}{l}\text { fakty do- } \\
\text { konane }\end{array}$ & $\begin{array}{l}\text { skończone fakty / } \\
\text { wykonane fakty / } \\
\text { zrealizowane fakty }\end{array}$ \\
\hline $\begin{array}{l}\text { ein fauler } \\
\text { Kompromiss } \\
\text { zweifelhafter Kom- } \\
\text { promiss / }\end{array}$ & $\begin{array}{l}\text { zgnity kom- } \\
\text { promis }\end{array}$ & $\begin{array}{l}\text { watpliwy kompromis / } \\
\text { zty kompromis }\end{array}$ \\
\hline
\end{tabular}

Häufig werden dabei aber auch Unterschiede zwischen den Sprachen offensichtlich. So heißt es beispielsweise im Deutschen schlechtes Gewissen, aber ungutes Gefühl. Im Polnischen wird dagegen das ,Gewissen' eher als ,unrein' bezeichnet (nieczyste sumienie) - zte sumienie, das etwas vorwirft und einen anklagt, tritt im Vergleich dazu viel seltener auf. ${ }^{1}$ In Bezug auf ,Gefühl' wird wiederum häufiger ,schlecht" verwendet - niedobre ,ungut' tritt im Vergleich

1 Das Nationalkorpus der Polnischen Sprache (im Folgenden - NKJP) liefert für złe sumienie lediglich 7 Kookkurrenzen, für nieczyste sumienie werden $123 \mathrm{Be}$ lege angeführt. 
zu złe przeczucie weit seltener auf. Einige weitere normativ festgelegte Unterschiede zeigt die folgende Tabelle:

\begin{tabular}{|c|c|c|c|}
\hline \multicolumn{2}{|l|}{ DEUTSCH } & \multicolumn{2}{|l|}{ POLNISCH } \\
\hline $\begin{array}{l}\text { normbedingte } \\
\text { Kombination / } \\
\text { Kollokation }\end{array}$ & $\begin{array}{l}\text { systembedingte / } \\
\text { alternative Aus- } \\
\text { drucksmöglichkeiten }\end{array}$ & $\begin{array}{l}\text { normbedingte } \\
\text { Kombination / } \\
\text { Kollokation }\end{array}$ & $\begin{array}{l}\text { systembedingte / } \\
\text { alternative Aus- } \\
\text { drucksmöglichkei- } \\
\text { ten }\end{array}$ \\
\hline $\begin{array}{l}\text { ein fauler } \\
\text { Kompromiss }\end{array}$ & $\begin{array}{l}\text { ein zweifelhafter } \\
\text { Kompromiss / } \\
\text { ein übler Kompro- } \\
\text { miss }\end{array}$ & $\begin{array}{l}\text { zgnity kompro- } \\
\text { mis }\end{array}$ & $\begin{array}{l}\text { wątpliwy kompro- } \\
\text { mis / } \\
\text { zty kompromis }\end{array}$ \\
\hline aber: & & aber: & \\
\hline faule Ausrede & $\begin{array}{l}\text { schlechte Ausrede / } \\
\text { üble Ausrede } \\
\text { schwache Ausrede }\end{array}$ & $\begin{array}{l}\text { kiepska } \\
\text { wymówka }\end{array}$ & $\begin{array}{l}\text { licha wymówka / } \\
\text { słaba wymówka } \\
\text { zła wymówka }\end{array}$ \\
\hline $\begin{array}{l}\text { der gebotene / } \\
\text { gebührende } \\
\text { Ernst }\end{array}$ & $\begin{array}{l}\text { der angemessene / } \\
\text { gehörige Ernst }\end{array}$ & $\begin{array}{l}\text { należyta / } \\
\text { należna powaga }\end{array}$ & $\begin{array}{l}\text { odpowiednia / } \\
\text { wtaściwa / sto- } \\
\text { sowna powaga }\end{array}$ \\
\hline aber: & & aber: & \\
\hline $\begin{array}{l}\text { blutiger / } \\
\text { bitterer Ernst }\end{array}$ & herber Ernst & $\begin{array}{l}\text { śmiertelna / } \\
\text { petna powaga }\end{array}$ & groźna powaga \\
\hline $\begin{array}{l}\text { blutige Trä- } \\
\text { nen weinen }\end{array}$ & $\begin{array}{l}\text { heftige Tränen wei- } \\
\text { nen / } \\
\text { starke Tränen weinen }\end{array}$ & $\begin{array}{l}\text { ptakać rzewnymi } \\
\text { tzami }\end{array}$ & $\begin{array}{l}\text { płakać rzęsistymi } \\
\text { tzami / płakać } \\
\text { obfitymi tzami }\end{array}$ \\
\hline $\begin{array}{l}\text { herbe / bittere } \\
\text { Enttäuschung }\end{array}$ & saure Enttäuschung & $\begin{array}{l}\text { gorzkie roz- } \\
\text { czarowanie }\end{array}$ & $\begin{array}{l}\text { cierpkie rozczaro- } \\
\text { wanie }\end{array}$ \\
\hline aber: & & aber: & \\
\hline $\begin{array}{l}\text { herbe / har- } \\
\text { sche / scharfe } \\
\text { Kritik }\end{array}$ & bittere Kritik & ostra krytyka & zaostrzona krytyka \\
\hline
\end{tabular}

Wie Coseriu zu Recht bemerkt, folgt aus derartigen normativ bedingten festen Kombinationen, die einzelsprachspezifisch sein können, der Schluss, man könne im Allgemeinen ,keine Sprache nur mit Kenntnis des Systems sprechen: eine Kenntnis der situationellen und kontextuellen Anwendungsnormen ist dazu ebenso notwendig“ (COSERIU 1970:43f.). Da Wörter „häufig in oft wiederholten Wortverbindungen“ vorkommen (STUBBS 1997:515) und diese von Sprache zu Sprache differieren können, erscheint es notwendig, Wörter in ihren syntagmatischen Zusammenhängen zu kennen und zu lernen. Der Sprachgebrauch beruht nämlich laut STUBBS (1997:152) darauf, dass die Sprachteilhaber stets auf vorgefertigte Mehrwort-Einheiten zurückgreifen: „Es wäre schlicht unmöglich, andere Sprecher überhaupt zu verstehen, wenn 
alles neu und kreativ wäre. Abertausende von solchen Mehrwort-Einheiten müssen als solche im Gedächtnis gespeichert sein." Ähnlich argumentiert diesbezüglich WILSS (1997:80f.): „Intersubjektive Verständigung wäre zweifellos sehr viel schwieriger, gäbe es nicht einen Vorrat an robusten Schemata oder an kommunikativen Handgriffen, die nur eine ,flache' Verarbeitungsebene erfordern [...].“ Den Grund für das Zurückgreifen auf vorgefertigte, feststehende Syntagmen und Textbausteine sieht WILSS (1997:70) darin, dass formelhafte, stereotype Sprache aus Gewohnheit verwandt wird: „Der Mensch ist ein Gewohnheitstier; er handelt, wo immer er kann (und manchmal auch da, wo dies nicht zulässig ist) nach dem Prinzip ,Consuetudo altera natura'; er neigt dazu, konfigurationsbetonte sprachliche Ausdrucksweisen unter vergleichbaren Gebrauchsbedingungen repetitiv einzusetzen [...].“

Das Problematische, das sich dabei insbesondere für den Fremdsprachenerwerb, aber auch für die Textproduktion und das Übersetzen in die Zweitsprache ergibt, gründet nicht so sehr auf der Qualität derartiger konventionalisierter Kombinationen als vielmehr auf deren Quantität (vgl. HAUSMANN 2007:229):

Wenn man sich klar macht, dass eine große Zahl banalster Alltagsaussagen kollokativ geregelt ist (Sagt man tun oder machen oder beides? Sagt man englisch broad oder wide oder beides mit und ohne Bedeutungsunterschied?) [...], dann wird klar, dass es sich um Zehntausende von Einheiten handelt. (HAUSMANN 2003:85)

Ähnlich sieht dies LUDEWIG (2005:177): „Die Anzahl der zu memorierenden Kollokationen ist enorm." Die Problematik verschärft sich darüber hinaus zum einen dadurch, dass die kollokativen Verbindungen varietätenspezifische Unterschiede aufweisen können. Allein aus diatopischer Sicht macht beispielsweise HEID (2011:534, 549f., 554) anhand einer Analyse von Zeitschriftenkorpora aus Deutschland (D), Österreich (AT), der Schweiz (CH) und Südtirol (ST) auf folgende Unterschiede aufmerksam:

D: Zuschuss geben - CH: Zustupf leisten,

D: Ermittlungen aufnehmen - CH: Erhebungen aufnehmen,

D: betrügerischer Bankrott - AT: betrügerische Krida,

D: niedriger Preis - CH: tiefer Preis,

D: feste Stelle - ST: fixe Stelle,

D: amtierender Weltmeister - AT: regierender Weltmeister,

D: einen Kredit bewilligen - CH: einen Kredit sprechen,

D: hohe Abfindung - AT: hohe Abfertigung,

D: (s)ein Auskommen finden - AT: das Auslangen finden. 
Felicja Księżyk

Zum anderen liegt die Schwierigkeit auch darin begründet, „,ass sich permanent neue Kollokationen etablieren“ (LUDEWIG 2005:176), wohingegen andere unüblich werden. Während es noch bei Goethe außer Zweifel sein, Vorschläge tun, auf etw. Verzicht tun hieß, so lauten ihre heutigen Entsprechungen außer Zweifel stehen, Vorschläge machen, auf etw. Verzicht leisten oder einfach verzichten auf etw. (vgl. BURGER 2003:135). Ähnliche Unterschiede werden bei der Sichtung älterer Zeitschriftenkorpora offenbar. Beim Vergleich von Zeitungen aus dem Ende des 18. Jahrhunderts mit dem heutigen Sprachgebrauch fallen beispielsweise folgende Abweichungen auf (nach BURGER 2003:136):

\begin{tabular}{|l|l|}
\hline um 1800 & Heute \\
\hline jmdm. Einhalt thun & jmdm. Einhalt gebieten \\
\hline Aufsehen machen & Aufsehen erregen \\
\hline eine Forderung an jmdn. thun & eine Forderung an jmdn. stellen \\
\hline Frieden machen mit jmdm. & Frieden schließen mit jmdm. \\
\hline
\end{tabular}

Daraus leitet Burger (2003:136) die Feststellung ab: „Im Vergleich mit der Situation von 1800 haben es die heutigen Sprecher und Schreiber schwerer: Statt eines kleinen Sets von Verben, die in Kollokationen mit Substantiven eintreten, müssen sie jeweils das spezifische (das ,treffende') Wort finden.“

\section{Wesensmerkmale von Kollokationen}

Der deutsche Romanist Hausmann, dem das Verdienst zugeschrieben wird, Kollokationen aus der Peripherie der Phraseologie ins Zentrum des Interesses gerückt zu haben (vgl. STEYER 1998:99), definiert Kollokationen als „Halbfertigprodukte der Sprache, welche der Sprecher nicht kreativ zusammensetzt, sondern als Ganzes aus der Erinnerung holt“ (HAUSMANN 1984:398f.). Auch wenn viele Kollokationsforscher gleiche oder ähnliche Merkmale bei der Bestimmung von Kollokationen festlegen, gibt es dennoch Unterschiede hinsichtlich dessen, was letztendlich bei ihnen als Kollokationen eingestuft und was ausgeblendet wird. Im Folgenden sollen die sich in der einschlägigen Literatur wiederholenden Merkmale, die auch für den vorliegenden Beitrag als Kriterien gelten, näher beleuchtet werden.

\subsection{Polylexikalität}

Das Kriterium der Polylexikalität, welches verlangt, dass eine Kollokation aus mehr als einem Wort besteht, gilt als unproblematisch, einigt man sich darüber, was unter einem ,Wort' verstanden wird (vgl. BURGER 2003:15 und 
KÜHTZ 2007:68). Nicht eindeutig wird allerdings die Frage beantwortet, ob es sich dabei um Autosemantika handeln muss oder auch Synsemantika kollokative Verbindungen eingehen können. Betrachtet Gładysz Kollokationen als „syntagmatische Wortverbindungen zweier, in seltenen Fällen dreier Autosemantika“" (GŁADYSZ 2003:48, vgl. auch LÜGER 2004:48 oder BISCHOF 2007:84) - und dieser Auffassung pflichte ich im vorliegenden Beitrag bei so versteht etwa FeILKE (2004:52) darunter auch synsemantische Kombinationen.

Problematisch kann das Kriterium der Polylexikalität im Sinne von graphischer Diskontinuität, jedoch nicht zuletzt auch im Sprachvergleich werden. Während das Deutsche nämlich eine kompositionsfreudige Sprache ist, ist im Polnischen häufiger eine Wortgruppenstruktur anzutreffen, wie die folgenden Beispiele veranschaulichen:

dt. Vollidiot - pl. skończony idiota,

dt. Scharfschütze - pl. strzelec wyborowy,

dt. Schaltjahr - pl. rok przestępny,

dt. Kalenderjahr - pl. rok kalendarzowy,

dt. Vorjahr - pl. ubiegly rok.

Als Kollokationskandidaten werden folglich in Anlehnung an KÜHTZ (2007:114) nur diejenigen Komposita berücksichtigt, welche eine parallele und funktional äquivalente Wortgruppenstruktur aufweisen, etwa Vorjahr $\rightarrow$ voriges Jahr.

Was die Polylexikalität anbelangt, bleibt noch zu erwähnen, dass binäre Strukturen dabei zwar den Ausgang bilden, sie können allerdings zu komplexen Kollokationen kombiniert werden und sich zu sogenannten Tripel- oder Quadrupel-Strukturen verbinden lassen, etwa:

Kritik üben + harsche Kritik $=$ harsche Kritik üben,

blutige Tränen + Tränen weinen = blutige Tränen weinen,

drastische Maßnahmen + Maßnahmen ergreifen = drastische Maßnah-

men ergreifen,

Vorschriften + Anwendung finden + entsprechende Anwendung $=$ Vorschriften finden entsprechende Anwendung. 
Felicja Księżyk

\subsection{Stabilität}

Als weiteres wesentliches Charakteristikum kollokativer Verbindungen gilt die Stabilität / Festigkeit / Fixiertheit bzw. idiomatische Prägung. ${ }^{2}$ Der Begriff ist nach Burger schwerer fassbar als die Polylexikalität. Ob eine Mehrwortverbindung diesem Kriterium gerecht wird, lässt sich nämlich lediglich aufgrund ihrer Gebräuchlichkeit entscheiden, und die Frage, ob eine Wortkombination als allgemein gebräuchlich aufgefasst werden kann, gestaltet sich schwierig in ihrer Beantwortung (vgl. BURGER 2003:16). Die Festigkeit einer Verbindung manifestiert sich auf mehreren Ebenen. Zum einen wird Festigkeit im strukturellen Sinne betrachtet. Damit ist gemeint, dass Kollokationen einerseits lexikalischen Restriktionen unterliegen: die Substituierbarkeit der Kollokationsbestandteile durch Synonyme ist kaum oder beschränkt möglich (vgl. KRATOCHVÍLOVÁ 2011:88), andererseits können aber Kollokationen morphosyntaktische Irregularitäten aufweisen, ,die über die normalen Regularitäten hinausgehen“ (BURGER 2003:20).

An folgenden Beispielen mit Zähnen, etwa Zähne putzen, Zähne fletschen, Zähne blecken, lässt sich erkennen, dass die verbalen Kollokatoren, was die lexikalischen Restriktionen angeht, nicht oder nur kaum substituierbar sind. Im Deutschen sind Zähne blecken und fletschen nur gegeneinander austauschbar, die polnische Entsprechung szczerzyć zęby weist keine Kommutationsmöglichkeiten auf. Als Alternative zu Zähne putzen werden zwar im Digitalen Wörterbuch der Deutschen Sprache (im Folgenden - DWDS) auch Zähne säubern und Zähne reinigen angeführt, etwa:

Er habe die falschen Zähne gesäubert und dann zum Trocknen auf die Fensterbank seines Hotels in Nordwales gelegt, wo er seine Ferien verbrachte. (Der Tagesspiegel, 09.07.2001)

Die Spange ist herausnehmbar - man kann die Zähne also wie gewohnt reinigen. (Berliner Zeitung, 26.08.2004)

Beide Verbindungen sind jedoch weit weniger gebräuchlich als Zähne putzen. Für Zähne säubern kommen nur 7 Belege vor, Zähne reinigen sind 22 Mal belegt, Zähne putzen ergibt 327 Treffer. ${ }^{3}$ Andere vom System her mögliche Kombinationen wie Zähne waschen sind nach DWDS nicht usuell.

Die eingeschränkte lexikalische Substituierbarkeit bzw. Produktivität spiegelt sich ferner im begrenzten Kombinationspotential der Kollokationspartner

2 Die idiomatische Prägung bzw. „morphosyntaktische Idiomatisierung“ wird dabei nicht im Sinne von idiomatisierter Bedeutung, sondern formaler Fixiertheit aufgefasst (vgl. HEID 2011:538f.).

3 Suchanfragen im DWDS (13.08.2013). 
wider. So verbinden sich blecken, fletschen und szczerzyć lediglich mit Zähnen, im Deutschen kommt vereinzelt nur noch Gebiss in Verbindung mit diesen Verben vor. Ähnliches lässt sich in Bezug auf abgedroschen, verheerend oder gravierend feststellen, die alle ebenfalls eine beschränkte Kollokabilität aufweisen, auch wenn sie bereits einige Kookkurrenzpartner haben. Typisch sind sie in den Verbindungen abgedroschene Phrasen, verheerende Folgen und gravierende Unterschiede. Analog weisen im Polnischen die Kollokatoren wyświechtany, druzgocacy oder wierutny ein enges Kombinationspotential auf, weshalb die Verbindungen wyświechtany frazes, druzgocaca klęska und wierutna bzdura ebenfalls als kollokativ gewertet werden können.

Die morphosyntaktische Eingeschränktheit von Kollokationen ist indes keine Conditio sine qua non für deren Status. Im Vergleich zu Idiomen sind Kollokationen nämlich weniger stark fixiert und lassen sich in einem höheren Grad transformieren (vgl. KONECNY 2010:113f.). Vielmehr geht es hierbei darum, dass das präferierte Vorkommen einiger Kombinationen in bestimmten morphosyntaktischen Formen oder eventuelle Verstöße der kanonischen Form von Kollokationen gegen die üblichen morphosyntaktischen Regularitäten die Kollokativität indizieren können (vgl. LUDEWIG 2005:93):

Kollokationen [stellen] [...] ein interessantes Phänomen im Grenzbereich zwischen Lexik und Grammatik dar, insofern sehr viele Kollokationen spezifische morphosyntaktische Eigenschaften aufweisen, etwa hinsichtlich des Artikelgebrauchs, der Modifizierbarkeit durch Adjektive oder Adverbien, bezüglich Präferenzen für das Auftreten im Aktiv oder im Passiv usw. (HEID 2011:536)

Als Beispiele dafür lassen sich etwa der artikellose Gebrauch bei Beschwerde einlegen oder Kritik üben anführen, die Pluralverwendung bei gemischte Gefühle oder Ermittlungen führen, letzteres im Gegensatz zu der Singularverwendung bei der polnischen Entsprechung prowadzić dochodzenie. Ebenso heißt es im Deutschen Kompromisse machen, aber nicht einen Kompromiss machen (vgl. HAUSMANN 2004:322). Sehr eingeschränkt ist beispielsweise auch die Morphosyntax der Kollokation in Kraft treten: Gebräuchlich ist nur die singularische, artikellose Form und nicht etwa in die Kräfte treten (vgl. HeID 2011:538f.). Bei den Verbindungen sich eine Blase laufen oder Geschmack an etw. finden ist es wiederum die „vom üblichen Gebrauch des verbalen Kollokationspartners abweichende Valenz“, die die Kollokativität dieser Kombinationen indiziert (vgl. LUDEWIG 2005:93f.). Wie bereits angedeutet, weisen nicht alle Kollokationen eine starke morphosyntaktische Fixiertheit auf. Anhand der Kollokation eine Rede halten soll im Folgenden veranschaulicht werden, dass sich Kollokationen vielfach morphosyntaktisch 
regulär verhalten und hinsichtlich der üblichen Transformationstests keinen Restriktionen unterliegen ${ }^{4}$ :

a) Variabilität im Numerus: Danach wird Wulff seine erste Rede als Staatsoberhaupt halten. / Er hält seine Reden auf Französisch, die schwedische Sprache meistert er nie, später tritt oft sein Sohn Oscar als Co-Redner und Übersetzer des Vaters auf.

b) Variabilität im Artikelgebrauch (definit und indefinit): Er hatte vor Guttenbergs verbaler Vorwärtsverteidigung eine Rede gehalten und gefordert, dass die Wehrpflichtigen sinnvolle Beschäftigungen erhalten. / Als David im Kampf gegen Goliath hielt er die üblichen Reden, Powerpoint-Präsentationen, wichtige Testemonials von wichtigen Leuten. / Merkel hat die Rede ihres Lebens gehalten.

c) Negierbarkeit des Substantivs: Danach hat Hitler keine öffentliche Rede mehr gehalten.

d) optionale Attribuierung durch Adjektive: Am Mittag hielt SPDFraktionschef Frank-Walter Steinmeier vor den Delegierten eine kämpferische Rede. / Oder auch wenn Falconer an der Universität eine improvisierte Rede über die verheerenden Folgen der Angst hält.

e) Komposition: Noch in der anstrengenden Schlussphase des Schreibens sprang er als Laudator für andere Autoren von vier- bis sechshundertseitigen Werken ein, hielt große Bundestagsreden, managte eine veritable Parteikrise - und schwitzte an seinem Aufstieg zum Marathonläufer, zwei Stunden täglich. Respekt, Joschka Fischer, und alles Gute zum Fünfzigsten.

f) Erweiterbarkeit durch Präpositionalphrasen oder genitivische Nominalphrasen: Strategische Reden zu Reformen hält er in anderer Umgebung, meist stehend vor größerem Publikum, das zwischendurch klatscht. / Er hielt eine Rede des Hasses.

g) Modifizierbarkeit durch einen Relativsatz: Und in ihrer Rede, die sie hielt, als sie 1978 den Friedenspreis des Deutschen Buchhandels entgegennahm $[\ldots]^{5}$

h) anaphorischer Bezug per Personalpronomen: Zum ersten Mal seit 43 Jahren erschien ein Moskauer Staatschef auf der Münchner Si-

4 Die Umformungsproben werden in Anlehnung an Ludewig (2005:95f.) angeführt. Beispielsätze mit der Kollokation eine Rede halten entstammen dem $D W D S$ oder der am Institut für deutsche Sprache in Mannheim konzipierten Textdatenbank Cosmas.

5 Quelle: http://lfs-berufskolleg-geldern.de/Lindgren.htm (16.08.2013). 
Die Routiniertheit des Sprachgebrauchs am Beispiel kollokativer Verbindungen

cherheitskonferenz; dann hielt er eine Rede, wie man sie im Westen seit Sowjetzeiten nicht mehr gehört hatte.

i) Erfragbarkeit: Was hat er gehalten, eine Rede? ${ }^{6}$

j) Variabilität des Verbs in Tempus, Numerus, Person und Modus: Hortensia Völckers, die künstlerische Leiterin der Bundeskulturstiftung, hält eine Rede voller Empathie. / „In einem Parlament der Vergangenheit hätten sie diese Rede nicht halten können“, sagte der CDU-Politiker.

k) Negierbarkeit des Verbs: Dann halte er die ganze Rede nicht. / Die die Gala zur Verleihung des Deutschen Fernsehpreises als »endlosen Unsinn« bezeichnet, nachdem sie dort die wichtigste Rede, nämlich die auf Marcel Reich-Ranicki, nicht halten durfte?

1) Nominalisierbarkeit: Gelernt hat Agnieszka Brugger das Halten von Reden schon vor ihrer Zeit als Abgeordnete. ${ }^{7}$

m) adverbiale Modifizierung: Wir halten nicht gern lange Reden.

n) Passivierung (Vorgangspassiv): Danach werden die üblichen Reden über Unabhängigkeit und ermächtigenden Stolz der eigenen Macht gehalten.

o) Bildung des Zustandspassivs: Die letzte Rede war gehalten, da trat der Präsident der Stiftung Preußischer Kulturbesitz, Klaus-Dieter Lehmann, noch einmal vor. ${ }^{8}$

p) Bildung des unpersönlichen Passivs: Kränze wurden niedergelegt, Gebete wurden gesprochen, es wurden Reden gehalten und sogar Graffiti von Mauerresten geschrubbt [...].

Neben der Festigkeit auf der strukturellen Ebene ist bei Kollokationen ähnlich wie bei Idiomen von psycholinguistischer Festigkeit auszugehen (vgl. BURGER 2003:17). Auch Kollokationen werden nämlich im mentalen Lexikon gespeichert und als Einheit abgerufen. Dies tritt besonders deutlich am Phänomen der „Versprecher“ zutage, die nicht nur FremdsprachensprecherInnen, sondern auch MuttersprachlerInnen unterlaufen, wie HÄCKI BUHOFER (2011:521) konstatiert:

6 Für die Erfragbarkeitsprobe konnte kein Beleg nachgewiesen werden, so dass der Beispielsatz konstruiert wurde.

7 http://www.frankenpost.de/jugend/kinderzeitung/wissen/hintergruende/Redenhalten-im-Bundestag;art6477,2762402 (16.08. 2013).

8 http://www.berliner-zeitung.de/archiv/bode-museum---gestern-abend-wurdeberlins-neuer-kunstpalast-mit-einem-festakt-eroeffnet--von-donnerstag-an-kanndie-vielleicht-beste-skulpturensammlung-der-welt-endlich-wiederentdecktwerden--der-bund-hat-es-gerichtet,10810590,10427744.html (16.08.2013). 
Felicja Księżyk

Das ist auch für native speakers von Bedeutung, weil sich in journalistischen und anderen Gebrauchstexten sehr viele fragliche Kollokationen bzw. falsch verwendete Kollokationen finden, wonach beispielsweise Hoffnungen auf jemandem lasten. Eine solche Äußerung könnte bei entsprechender Absicht eine aussagekräftige Variante zu Hoffnungen, die auf jmdm. ruhen bzw. eine ebenso aussagekräftige intendierte Kontamination von Hoffnung, die ruht, und Verantwortung, die lastet, darstellen.

So weisen beispielsweise die folgenden (authentischen) Äußerungen einige Ungereimtheiten und Unregelmäßigkeiten auf:

1. Dieses Motiv lässt Parallelen zu Platons Höhlengleichnis aufstellen.

2. Der Bau dieser Kapelle ist von einer Legende umhüllt.

3. [...] eine Idee, die gut in der philosophischen Tradition verankert ist $[\ldots]$.

4. Wir und die Stipendiaten erweisen die Bereitschaft, Sie in Ihrer seelsorgerischen Arbeit, besonders mit den Jugendlichen, zu helfen.

In 1 wurde der Verbkollokator falsch gewählt. Zwar heißt es Thesen / Behauptungen / Theorien aufstellen. Parallelen werden allerdings der Norm nach gezogen. 2 zeigt, dass der Sprecher zwei kollokative Verbindungen verwechselt hat. Zwar heißt es im DWDS „das alles bleibt wohl immer von einem Geheimnis umhülltt ${ }^{\star 9}$, typischerweise sagt man jedoch in Bezug auf Legenden: von Legenden umrankt sein. Bei 3 ist es zwar möglich, gut und verankert miteinander zu verbinden, weit typischer ist allerdings die Kombination fest verankert.

Von grammatischen Verstößen in 4 abgesehen, sticht hier besonders die Kombination Bereitschaft erweisen als ungewöhnlich heraus. Während erweisen in Verbindung mit Ehre und Dienst typisch ist, würde man in diesem Zusammenhang Bereitschaft bekunden oder erklären erwarten, oder noch einfacher sich bereit erklären. Unmarkiert dürfte diese Äußerung also lauten: Wenn wir Sie auf irgendwelche Weise bei der seelsorgerischen Arbeit, insbesondere mit jungen Menschen, unterstützen könnten, wären wir dazu gerne bereit.

Die psychologische Festigkeit von Kollokationen ist eng gekoppelt an ein weiteres Kriterium, das an diese Verbindungen angelegt wird, nämlich das der Assoziativität.

9 http://www.dwds.de/?qu=umh\%C3\%BCllen\&submit_button=Suche \&view=1 (16.08.2013). 
Die Routiniertheit des Sprachgebrauchs am Beispiel kollokativer Verbindungen

\subsection{Assoziativität / Evozierbarkeit / Erwartbarkeit}

Assoziative Beziehungen kennzeichnen „nicht nur Kollokationen, sondern auch Wörter, die zu einem Wortfeld gehören, Antonyme, Bestandteile eines Phraseologismus“ (GŁADYSZ 2003:61). Im Gegensatz dazu stellen Kollokationen allerdings lexikalische Syntagmen dar. Die Assoziativität von Kollokationen beruht darauf, ,dass ein Ausdruck seinen Kollokationspartner gewissermaßen zwangsläufig fordert“ (HÄCKI BUHOFER 2011:522). Anders ausgedrückt: „,bei Erwähnung einer ihrer Komponenten fällt einem kompetenten Sprachteilhaber die andere ein“ (GŁADYSZ 2003:49). Die Evozierbarkeit der Kollokationen ist gegenseitig gerichtet, allerdings kann „,der Grad der Assoziativität in verschiedenen Richtungen verschieden stark" sein (GŁADYSZ 2003:62). Die Verben blecken / fletschen bzw. szczerzyć im Polnischen werden von den Sprechern des Deutschen bzw. Polnischen automatisch mit Zähnen assoziiert, umgekehrt evozieren jedoch Zähne nicht unbedingt in erster Linie blecken oder fletschen, da das Substantiv über ein umfangreicheres Kombinationspotential verfügt - allein in der syntaktischen Funktion eines Aktivsubjekts liefert das $D W D S$ folgende Kombinationsmöglichkeiten:

,Zahn' ist Aktivsubjekt von
abgebrochen angegriffen angenagt anhaben aufweisen ausbeißen ausfallen
aussehen behandeln beißen bilden blecken bleiben blitzen brechen dienen
durchbrechen entwickelt erhalten fallen faulen fehlen fletschen fliegen
frisst führen gehören geschafft halten hindeuten hingen hinterlassen klap-
perten knirschen kosten lassen leiden leuchten liegen machen nachwach-
sen nagt putzen ragen richten schimmerten schlagen schmerzt sitzen
stammen stecken stehen strahlen trafen tropft tun verfault verloren verra-
ten verschwanden wachsen wackeln wirken zeigen zerstört ziehen zuge-
setzt zusammenbeißen

Dabei stellt sich heraus, dass einige der hier angeführten Kombinationsmöglichkeiten syntaktisch nicht zusammengehörig sind ${ }^{10}$ bzw. in anderen syntak-

10 Dass die Basis Zahn nicht immer mit den angeführten Verben in syntagmatischer Verbindung steht, veranschaulichen etwa die folgenden Belege: „Der Zahn hatte sich entzündet, die Entzündung auf den Schädel übergegriffen und zur Erblindung des Tieres geführt.“ (Die Zeit, 26.04.2007) oder „Nicht erst duschen und Zähne putzen, dabei fallen Ihnen 14 Ausreden ein." (Berliner Zeitung, 21.11.2003). 
Felicja Księżyk

tischen Funktionen weitaus häufiger begegnen, teilweise aber die syntaktische Zuordnung sogar fehlerhaft ist. ${ }^{11}$

Aus der Analyse der Kombinationspotentiale einzelner Lexeme wird allerdings ersichtlich, dass der „Grad der Assoziativität [...] umgekehrt proportional zum Umfang des Kollokationspotentials [ist]“ (GŁADYSZ 2003:62). Mit der Assoziativität ist auch die gegenseitige Voraussagbarkeit bzw. Prädikabilität der Kollokationsbestandteile verbunden. Kommen die Verben blecken oder fletschen vor, so ist die Wahrscheinlichkeit, dass in Verbindung damit auch das Substantiv Zähne gebraucht wird, sehr hoch. Diese Eigenschaft wird in der Computerlinguistik genutzt, indem statistische Assoziationsmaße eingesetzt werden: „Wenn zwei Wörter proportional häufiger miteinander vorkommen als statistisch erwartbar, dann werden sie von den Assoziationsmaßen identifiziert und extrahiert" (HEID 2011:539). Assoziationsmaße werden anhand unterschiedlicher Tests zur Feststellung der kollokationellen Signifikanz von Zweierkombinationen eingesetzt. „Ein Bigramm gilt dann als signifikant, wenn seine Frequenz im Korpus höher ist als die Frequenz seiner Einzelkomponenten“ (KRATOCHVÍlOVÁ 2011:187). Im DWDS, das diesem Beitrag zugrunde lag, kommen mehrere Assoziationsmaße zur Anwendung, u. a. der Mutual Information-Test (MI) und der logDice-Score. Das Assoziationsmaß $\log$ Dice weist gegenüber der Mutual Information einige Vorteile auf, u.a. scheint es ,besser geeignet [...], Assoziationsmasse zwischen verschiedenen Korpora zu vergleichen“ (LUDER 2011:116). Das theoretische Maximum des $\operatorname{logDice}$-Scores beträgt 14 Punkte, wobei nach LUDER (2011:117),,[ü]bliche Werte für gute Kollokationskandidaten [...] weniger als 10 Punkte [betragen]“. Bei Zähne fletschen liegt der mittels logDiceScore ermittelte Assoziationswert im DWDS bei 8,79, bei Zähne blecken beläuft er sich auf 7,89. Die freie Verbindung Zähne sehen aus weist eine Assoziationsstärke von 0,87 auf. $^{12}$

11 So verbindet sich etwa blecken mit Zahn häufiger in der Objektfunktion, allerdings führt das $D W D S$ auch Belege mit Subjektfunktion an: „Die grausam spitzen Zähne blecken und die winzigen Augen stieren kühl und teilnahmslos.“ (Die Zeit, 15.02.2006)

„Zweiunddreißig Zähne bleckten auf dem Papier.“ In: Renate Rasp (1967): Ein ungeratener Sohn. Köln, 140.

Bei fletschen oder zusammenbeißen treten lediglich Belege mit Zahn in der Objektfunktion auf, eventuell kommt es vor, dass die ganze Wortverbindung als Aktivsubjekt fungiert: „Die Zähne zusammenbeißen und den Gürtel enger schnallen, das ist jetzt seine Devise.“ (Die Zeit, 27.06.1986).

12 Suchanfrage im DWDS (16.08.2013). 
Die Routiniertheit des Sprachgebrauchs am Beispiel kollokativer Verbindungen

\subsection{Konventionalität}

Wie bereits eingangs dargelegt wurde, handelt es sich bei Kollokationen um Erscheinungen der Sprachnorm. Ihre Restringiertheit kommt dadurch zustande, dass sie „durch die Sprachgemeinschaft im Konventionalisierungsprozess akzeptiert" und dadurch fixiert werden (KRATOCHVÍLOVÁ 2011:95, vgl. auch STUBBS 1997:155). Die Kollokativität von Mehrwortverbindungen kann unterschiedliche Gründe haben. Zum einen kann es sich bei Kollokationen um Wortkombinationen handeln, die aufgrund der außersprachlichen Realität oder Semantik kookkurrieren. Mit der Änderung von sprachunabhängigen Gegebenheiten gehen auch sprachliche Änderungen einher, auch wenn letztere etwas hinterherhinken können. So heißt es zwar im Polnischen heute primär wybierać numer (die (Telefon-)Nummer wählen), wohingegen seinerzeit die Telefonnummer ,gedreht" wurde (wykręcać numer). Analog ist es im Deutschen, wo wählen das Verb drehen nahezu verdrängte. Im Falle von odkręcać kran (auch den Hahn aufdrehen) hat sich das Verb allerdings noch erhalten, auch wenn bei der bezeichneten Tätigkeit mittlerweile häufig kein Aufdrehen mehr erfolgt.

Zum anderen handelt es sich bei Kollokationen allerdings auch um Wortkombinationen, die ,durch den Usus überdurchschnittlich prädestiniert sind, miteinander vorzukommen“ (HÄCKI BUHOFER 2011:521):

Von einer kollokativen Wortverbindung kann man dann sprechen, wenn ein Sachverhalt durch mehrere Wortkombinationen ausgedrückt werden kann, aber von den SprecherInnen/SchreiberInnen häufig ein und dieselbe gewählt wird, bzw. beim Sprechen oder Schreiben von fremdsprachigen SprecherInnen auch bei Berücksichtigung der Bedeutungen der Einzelwörter eine unübliche Wortverbindung entsteht, weil sie die kollokative Wortverbindung nicht kennen. Analoges gilt auch in der Erstsprache, wenn es um professionelle bzw. professionalisierte Ausdrucksweisen geht. (HÄCKI BUHOFER 2011:522)

Beispiele, die die Konventionalität der Kollokationen veranschaulichen, finden sich im einleitenden Abschnitt, der kollokative Verbindungen als Erscheinungen der Sprachnorm darstellt.

\section{5 (Halb-)Kompositionalität}

Als eines der wichtigsten Merkmale der Kollokationen wird deren Halbkompositionalität oder Kompositionalität angesehen. Gemeint ist damit die Frage ihrer semantischen Transparenz. In dieser Hinsicht überwiegt in der Forschung der Standpunkt, dass sich die Bedeutung von Kollokationen entweder summarisch aus der Bedeutung ihrer Konstituenten herleiten lässt oder aber, dass Kollokationen aus semantisch ungleichgewichtigen Elementen bestehen: 
„Basen haben in der Kollokation dieselbe Bedeutung wie außerhalb, während Kollokatoren eine kollokationstypische Bedeutung haben.“ (HEID 2011:534) Die halbkompositionellen Verbindungen werden dabei als prototypischere Vertreter von Kollokationen betrachtet. Somit nehmen Kollokationen auf dem Kontinuum zwischen freien und idiomatischen Kombinationen eine Zwischenposition ein. In der folgenden Tabelle werden Kombinationen mit semantisch transparenten Kollokationen exemplifiziert, bei denen aufgrund von äquivalenten Entsprechungen im Deutschen und Polnischen keine Interferenzgrundlage vorliegt:

\begin{tabular}{|l|l|}
\hline Kollokation im Deutschen & Kollokation im Polnischen \\
\hline gemeine Lüge & podłe kłamstwo \\
\hline dreiste Lüge & bezczelne kłamstwo \\
\hline stiller Verehrer & cichy wielbiciel \\
\hline gehütetes Geheimnis & strzeżona tajemnica \\
\hline
\end{tabular}

Vielfach lassen sich allerdings Disparitäten im kollokationellen Usus des Deutschen und Polnischen feststellen, die zu Problemen führen können. In der folgenden Übersicht wird eine Beispielsammlung mit halbkompositionellen deutschen Kollokationen angeführt, die im Polnischen keine wörtlichen Entsprechungen haben. Die Problemstelle wird in der dritten Spalte angedeutet, in der die wahrscheinliche Übersetzung des Kollokators als potentielle Interferenz angeführt wird.

\begin{tabular}{|l|l|l|}
\hline $\begin{array}{l}\text { Kollokation im Deut- } \\
\text { schen }\end{array}$ & $\begin{array}{l}\text { Kollokation im Polni- } \\
\text { schen }\end{array}$ & /Interferenz/ \\
\hline bunte Auswahl & duży wybór & /groß/ \\
\hline faule Ausrede & kiepska wymówka & /miserabel, schlecht/ \\
\hline handfeste Beweise & $\begin{array}{l}\text { niezbity dowód / niezbite } \\
\text { dowody }\end{array}$ & $\begin{array}{l}\text { /ungeschlagen, durch- } \\
\text { schlagend/ }\end{array}$ \\
\hline abgedroschene Phrase & wyświechtany frazes & /abgewetzt; abgetragen/ \\
\hline stichhaltiger Beweis & przekonujący dowód & /überzeugend/ \\
\hline heikle Frage & drażliwe pytanie & /empfindlich/ \\
\hline dünner Tee & staba herbata & /schwach/ \\
\hline verheerende Folgen & fatalne skutki & /fatal/ \\
\hline blühende Phantasie & bujna wyobraźnia & /üppig/ \\
\hline lebhafte Diskussion & rozgorzata dyskusja & /entfachte/ \\
\hline nackte Tatsachen & suche fakty & /trocken// \\
\hline dunkles Geheimnis & mroczna tajemnica & /düster/ \\
\hline Wurzeln schlagen & zapuszczać korzenie & /wachsen lassen/ \\
\hline das Geheimnis lüften & zdradzić tajemnicę & /verraten/ \\
\hline $\begin{array}{l}\text { die Diskussion abbre- } \\
\text { chen }\end{array}$ & ucią́ dyskusję & /abschneiden/ \\
\hline
\end{tabular}


Die Routiniertheit des Sprachgebrauchs am Beispiel kollokativer Verbindungen

\begin{tabular}{|l|l|l|}
\hline Witze reißen & opowiadać kawaty & /erzählen/ \\
\hline $\begin{array}{l}\text { stockender/ruhender } \\
\text { Verkehr }\end{array}$ & ruch spowolniony & /verlangsamt/ \\
\hline verkrustete Strukturen & skostniate struktury & /verknöchert/ \\
\hline heller Wahnsinn & czyste szaleństwo & /rein/ \\
\hline helle Aufregung & jaskótczy niepokój & /schwalbenartig/ \\
\hline helle Freude & dzika radość & /wild/ \\
\hline betretenes Schweigen & kłopotliwe milczenie & /schwierig/ \\
\hline ein Nickerchen machen & uciąć sobie drzemkę & /abschneiden/ \\
\hline
\end{tabular}

Die obigen Auflistungen machen zudem deutlich, dass einige Kollokatoren ,in serieller Verknüpfung“ vorkommen, d.h. „stark reihenbildend wirken“ (KRATOCHVÍlOVÁ 2011:125f.), etwa: hell + Wahnsinn / Aufregung / Freude oder faul + Ausrede / Kompromiss.

\section{Fazit}

Wie Häcki Buhofer feststellt, sind „Kollokationen einer Sprache [...] ein fester Bestandteil der alläglichen Kommunikation. Sie werden beim Sprechen bzw. Schreiben nicht ad hoc gebildet, sondern als zusammengehörige Syntagmen abgerufen. Das hat zur Folge, dass alternative, frei kombinierte Konstruktionen [...] als unpassend empfunden werden, obwohl sie semantisch und syntaktisch ebenso korrekt wären“ (HÄCKI BUHOFER 2011:506). Für Muttersprachlerinnen und Muttersprachler sind Kollokationen in der Regel unauffällig, auffallend sind für sie jedoch die sogenannten Versprecher, bei denen gegen den konventionalisierten, routinemäßigen Sprachgebrauch verstoßen wird. Wegen der einzelsprachlichen Spezifik von Kollokationen und der damit einhergehenden Unvorhersehbarkeit, Idiosynkrasie und Arbitrarität scheitern allerdings Fremdsprachenlernende oft an ihnen (vgl. PÖLL 2002:84). Einerseits bestehen nämlich zahlreiche zwischensprachliche Unterschiede, andererseits gibt es auch kollokative Übereinstimmungen, so dass Fremdsprachenlernende sowohl die Abweichungen als auch die Gemeinsamkeiten memorieren müssen. Eine weitere Schwierigkeit gründet sich darauf, dass bei Kollokationen variationslinguistische Unterschiede bestehen und dass sie, wie Sprache überhaupt, einem steten Wandel unterliegen. In Bezug auf den interlingualen Vergleich ist ferner zu berücksichtigen, dass der Kollokation in einer Sprache nicht notwendig eine kollokative Verbindung in einer anderen Sprache entsprechen muss (vgl. BISCHOF 2007:21), wie die folgende Tabelle ansatzweise veranschaulicht: 
Felicja Księżyk

\begin{tabular}{|l|l|}
\hline dociekliwy czytelnik (Kollokation) & $\begin{array}{l}\text { interessierter Leser (freie Wortkombina- } \\
\text { tion) }\end{array}$ \\
\hline ponieść fiasko (Kollokation) & Schiffbruch erleiden (Idiom) \\
\hline Lästerungen ausstoßen (Kollokation) & bluźnić (einfaches Verb) \\
\hline
\end{tabular}

Des Weiteren ist zu beachten, dass aufgrund von Übereinstimmungen eventuelle direkte Übertragungen in eine Fremdsprache zwar als wörtliche Wiedergaben akzeptabel sein können, allerdings kann sich dabei zeigen, dass sie „Umgewichtungen in der Frequenzverteilung von Wortkombinationen“ bedingen (LUDEWIG 2005:168). So wären schlechtes Gewissen, ungutes Gefühl, nackte Tatsachen gegebenenfalls auch wörtlich ins Polnische zu übersetzen, analog ließe sich kiepska wymówka und śmiertelna powaga kompositionell ins Deutsche übertragen, dies wären jedoch nicht die typischsten Kombinationen, wie die Frequenzangaben (aus dem DWDS und dem NKJP) in der folgenden Tabelle bezeugen.

\begin{tabular}{|l|l|l|}
\hline $\begin{array}{l}\text { Kollokation in der Aus- } \\
\text { gangssprache }\end{array}$ & wörtliche Übertragung & $\begin{array}{l}\text { Kollokation in der } \\
\text { Zielsprache }\end{array}$ \\
\hline kiepska wymówka & schlechte Ausrede (38) & faule Ausrede (250) \\
\hline śmiertelna powaga & tödlicher Ernst (172) & $\begin{array}{l}\text { bitterer Ernst (353), } \\
\text { blutiger Ernst (226) }\end{array}$ \\
\hline schlechtes Gewissen & złe sumienie (7) & nieczyste sumienie (99) \\
\hline ungutes Gefühl & niedobre przeczucie (17) & zte przeczucie (62) \\
\hline nackte Tatsachen & $\begin{array}{l}\text { nagie fakty (11), } \\
\text { gote fakty (6) }\end{array}$ & suche fakty (25) \\
\hline
\end{tabular}

Abschließend lässt sich festhalten, dass die sprachliche Kommunikation in hohem Maße auf musterhaften, reproduzierbaren, normbedingten Formulierungen beruht. Dieser Befund resultiert aus korpuslinguistischen Untersuchungen, bei denen statistisch nachweisbar ist, wie hoch der Anteil vorgefertigter Äußerungen ist (vgl. STUBBS 1997:152): „mit Hilfe von großen Korpora [lassen sich nämlich - F.K.] das tatsächlich Realisierte und die immer wieder wiederholten Muster im tatsächlichen Sprachgebrauch [...] untersuchen“ (STUBBS 1997:155). Daraus wird mehrfach in der Forschung die Schlussfolgerung gezogen, dass im Sprachgebrauch die Routiniertheit über die Freiheit dominiere. So konstatiert etwa HAUSMANN (1995:19): „Im Wortgebrauch überwiegt die Bindung über die Freiheit, das Syntagmatische über das Paradigmatische, die Idiomatik über die Kompositionalität“. Ähnlich sieht es auch J. Sinclair, ein Vertreter des Britischen Kontextualismus, einer Schule, die die Kollokationsforschung in Gang gebracht hat. Sinclair ging von zwei Prinzipien der Sprachbeschreibung im Bereich der Lexik aus, dem open-choice principle und dem idiom principle. Während bei dem Prinzip 
der offenen Wahl ein Text als Ergebnis von zahlreichen Wahlmöglichkeiten betrachtet wird, beruht das idiomatische Prinzip darauf, dass der Sprachteilhaber zwischen halbvorgefertigten Phrasen als Versatzstücken auswählt. Obwohl in den meisten linguistischen Beschreibungen das open-choice principle dominiere, gebühre dem idiomatischen Prinzip eine weit wichtigere Rolle, als bisher angenommen (vgl. SinCLAIR 1987:320f.). Notwendig erscheint es folglich, der Kollokativität der Sprache größere Beachtung zu schenken, da diese keineswegs mit Phrasendrescherei gleichzusetzen ist, dadurch werden keine leeren, abgedroschenen Redewendungen geäußert:

$\mathrm{Da}$ wir triftige Gründe oder dunkle Ahnungen haben, daß wir die nackte Wahrheit wissen wollen, vor vollendeten Tatsachen stehen, daß wir berechtigte Zweifel haben, daß unser gesunder Menschenverstand uns sagt, daß die Lage ernst und der Zustand katastrophal ist [- das mögen zwar keine besonders kreativen, sondern stark routinierte Ausdrucksweisen sein - F. K.] [...], aber: sie erfüllen ihren kommunikativen Zweck, und sie haben sich - sicher nicht ganz zufällig im Lexikon einen festen Platz erobert. (STEIN 1995:92)

Obwohl die Routiniertheit im Sprachgebrauch oft im Oppositionsverhältnis zur Kreativität betrachtet wird (vgl. STEIN 1995:123), handelt es sich bei Kollokationen in der Tat um keine erstarrten, unveränderlichen Formulierungen, wie der Beitrag zu zeigen versuchte. Vielmehr ist anzunehmen, dass bei der Konventionalisierung und Etablierung mancher Kollokationen ursprünglich freie, kreativ zusammengesetzte Wortverbindungen durch Wiederholung ihre Kollokativität erlang(t)en.

\section{Literatur}

BISCHOF, BEATRICE-BARBARA (2007): Französische Kollokationen diachron. Eine korpusbasierte Analyse: http://elib.uni-stuttgart.de/opus/volltexte/2008/3418/pdf/ Franzoesische_Kollokationen.pdf (05.08.2013).

Burger, Harald (1998 / 2003): Phraseologie. Eine Einführung am Beispiel des Deutschen. Berlin.

BUtTlER, DANUTA (1982): Zagadnienia poprawności leksykalno-semantycznej. [Probleme der lexikalisch-semantischen Korrektheit]. In: ButTler, DanUtA / KuRKOWSKA, HALINA / SATKIEWICZ, HALINA (eds.): Kultura języka polskiego. Zagadnienia poprawności leksykalnej (Stownictwo rodzime). Warszawa, 44-228.

Coseriu, Eugenio (1970): Einführung in die strukturelle Betrachtung des Wortschatzes. Tübingen (=Tübinger Beiträge zur Linguistik 14).

- (1973): Probleme der strukturellen Semantik. Vorlesung gehalten im Wintersemester 1965/66 an der Universität Tübingen. Autorisierte und bearbeitete Nachschrift von Kastovsky. Tübingen (=Tübinger Beiträge zur Linguistik 40). 
ENgelberg, Stefan / Hollter, Anke / Proost, Kristel (eds.) (2011): Sprachliches Wissen zwischen Lexikon und Grammatik. Berlin/Boston.

FeILKe, Helmuth (2004): Kontext - Zeichen - Kompetenz. Wortverbindungen unter sprachtheoretischem Aspekt. In: STEYER, KATHRIN (ed.): Den Nagel auf den Kopf treffen. Wortverbindungen - mehr oder weniger fest. Berlin/New York, 41-64.

GŁADYSZ, MAREK (2003): Lexikalische Kollokationen in deutsch-polnischer Konfrontation. Frankfurt (M.).

HÄCKI BUHOFER, ANNELIES (2011): Lexikographie der Kollokationen zwischen Anforderungen der Theorie und der Praxis. In: Engelberg / Hollter / Proost, 505-531.

HAUSMANN, FRANZ JOSEF (1984): Wortschatzlernen ist Kollokationslernen. Zum Lehren und Lernen französischer Wortverbindungen. In: Praxis des neusprachlichen Unterrichts 31/4:395-406.

- (1995): Von der Unmöglichkeit der kontrastiven Lexikologie. In: KROMANN, HANSPEDER / KJER, ANNE LISE (eds.): Von der Allgegenwart der Lexikologie: kontrastive Lexikologie als Vorstufe zur zweisprachigen Lexikographie. Tübingen (=Lexicographica: Series maior 66), 19-23.

- (2003): Kollokationen in der Fachsprache: Schwerpunkt Französisch. In: JuNG, Udo O.H. / KolesniKova, ANGelina (eds.): Fachsprachen und Hochschule. Forschung-Didaktik-Methodik. Frankfurt (M.) (=Bayreuther Beiträge zur Glottodidaktik 9), 84-92.

- (2007): Die Kollokationen im Rahmen der Phraseologie - Systematische und historische Darstellung. In: Zeitschrift für Anglistik und Amerikanistik 55/3:217-234.

HEID, UlRICH (2011): Korpusbasierte Beschreibung der Variation bei Kollokationen: Deutschland - Österreich - Schweiz - Südtirol. In: ENGELBERG / Hollter / ProOst, 533-557.

KONECNy, CHRISTINE (2010): Kollokationen. Versuch einer semantisch-begrifflichen Annäherung und Klassifizierung anhand italienischer Beispiele. München (=Forum Sprachwissenschaften 8).

KratochvílovÁ, Iva (2011): Kollokationen im Lexikon und im Text. Mehrwortverbindungen im Deutschen und Tschechischen. Berlin (=Studien und Quellen zur Sprachwissenschaft 2).

KÜHTZ, STEFAN (2007): Phraseologie und Formulierungsmuster in medizinischen Texten. Tübingen (=Forum für Fachsprachenforschung 74).

LUDER, MARC (2011): Konstruktionen im Lexikon - Konstruktionen in der Erzählanalyse: http://www.zora.uzh.ch/60813/1/Dissertation_Luder.pdf (17.03.2014).

Ludewig, Petra (2005): Korpusbasiertes Kollokationslernen. Computer-Assisted Language Learning als prototypisches Anwendungsszenario der Computerlinguistik. Frankfurt (M.).

LÜGER, HeINZ-Helmut (2004): Kollokationen - zwischen Arbitrarität und Kompositionalität. In: POHL, InGE / KONERDING, KLAus-PETER (eds.): Stabilität und Flexibili- 
Die Routiniertheit des Sprachgebrauchs am Beispiel kollokativer Verbindungen

tät in der Semantik: Strukturelle, kognitive, pragmatische und historische Perspektiven. Frankfurt (M.) u. a., 45-66.

PÖLL, BERNHARD (2002): Spanische Lexikologie. Eine Einführung. Tübingen.

SINCLAIR, JOHN MCHARDY (1987): Collocation: a progress report. In: STEELE, Ross / THREADGOLD, TERRY (eds.): Language Topics. Essays in honour of Michael Halliday. Volume II. Amsterdam/Philadelphia, 319-331.

STEYER, KATHRIN (1998): Kollokationen als zentrales Übersetzungsproblem - Vorschläge für eine Kollokationsdatenbank Deutsch-Französisch auf der Basis paralleler und vergleichbarer Korpora. In: BRESSON, DANIEL (ed.): Lexikologie und Lexikographie Deutsch-Französisch. Aix-en-Provence (=Cahiers d'Études Germaniques 35), 95-113.

STEIN, STEPHAN (1995): Formelhafte Sprache. Untersuchungen zu ihren pragmatischen und kognitiven Funktionen im gegenwärtigen Deutsch. Frankfurt (M.)/Berlin/Bern u. a. (=Sprache in der Gesellschaft 22).

Stubbs, Michael (1997): „Eine Sprache idiomatisch sprechen“: Computer, Korpora, kommunikative Kompetenz und Kultur. In: MATTHEITER, Klaus J. (ed.): Norm und Variation. Frankfurt (M.)/Berlin/Bern u. a. (=Forum angewandte Linguistik 32), 151167.

Wilss, Wolfram (1997): Adjektiv/Substantiv-Kollokationen. Ein Beitrag zum Verständnis von Textbausteinen. In: Grbić, NADJA / Wolf, Michaela (eds.): Text Kultur - Kommunikation. Translation als Forschungsaufgabe. Festschrift aus Anla $\beta$ des 50jährigen Bestehens des Instituts für Übersetzer- und Dolmetscherausbildung an der Universität Graz. Tübingen (=Studien zur Translation 4), 67-82. 